Gláucia Rosana Guerra Benute'

Daniele Nonnenmacher ${ }^{2}$

Rosel Mieko Yamamoto Nomura ${ }^{3}$

Mara Cristina Souza de Lucia ${ }^{4}$

MARCelo Zugaib ${ }^{5}$

Artigo Original

Palavras-chave

Aborto induzido

Saúde da mulher

Aborto/legislação \& jurisprudência Atenção à saúde

Keywords

Abortion, induced

Women's health

Abortion/legislation \& jurisprudence Health care (Public Health)

\section{Influência da percepção dos profissionais quanto ao aborto provocado na atenção à saúde da mulher}

\author{
Perception influence of professionals regarding unsafe in attention to \\ women's bealth
}

\section{Resumo}

OBJETIVO: Identificar o conhecimento e a percepção dos profissionais da saúde em relação à legislação brasileira sobre o aborto provocado. MÉTODOS: Envelopes selados não identificados contendo os questionários foram enviados a todos os profissionais ( $n=149$ ) que trabalham no Departamento de Obstetrícia de hospital universitário e de hospital público da periferia de São Paulo. Responderam ao questionário 119 profissionais. Para análise dos dados, utilizou-se intervalo de confiança de 0,05 e os testes exatos de Fischer e $\chi^{2}$. RESULTADOS: Dos profissionais entrevistados, 48,7\% eram médicos, 33,6\% profissionais da área de enfermagem e 17,6\% eram profissionais de outras áreas (psicólogos, nutricionistas, fisioterapeutas, administrativos e técnicos de laboratórios). Constatou-se diferença significativa $(p=0,01)$ na proporção de profissionais que acreditam que o aborto por malformação fetal não letal e no aborto decorrente de gestações não planejadas deveriam ser incluídos na legislação brasileira. Observou-se que o conhecimento da legislação e da descrição das situações permitidas por lei acerca do aborto foi significativamente diferente na comparação entre os profissionais de saúde $(p=0,01)$. Quando questionados sobre as situações em que a legislação brasileira permite o aborto, observou-se que 32,7\% dos médicos, 97,5\% profissionais da área de enfermagem e 90,5\% dos demais profissionais desconhecem a legislação vigente. CONCLUSÃO: Neste estudo, evidenciou-se o desconhecimento dos profissionais de saúde com relação à legislação brasileira, em menor proporção entre obstetras e em maior proporção entre os profissionais da área de enfermagem. Foram constatadas atitudes de discriminação, julgamento e preconceito na assistência prestada às mulheres que provocam o aborto.

\section{Abstract}

PURPOSE: To identify the knowledge and awareness of health professionals regarding the Brazilian legislation on induced abortion. METHODS: Unidentified sealed envelopes containing the questionnaires were sent to all professionals ( $n=149$ ) working in the Obstetrics Department of a university hospital and public hospital at the periphery of São Paulo (SP), Brazil. A total of 119 professionals responded to the questionnaire. The 0.05 confidence interval and the Fisher exact test and $\chi^{2}$ test were used for data analysis. RESULTS: Of the respondents, $48.7 \%$ were physicians, $33.6 \%$ were nursing professionals and $17.6 \%$ were professionals from other fields (psychologists, nutritionists, physiotherapists, laboratory technicians and administrators). There was a significant difference ( $p=0.01$ ) in the proportion of professionals who believe that abortion for non-lethal fetal malformation and due to unplanned pregnancies should be included in the Brazilian legislation. It was observed that the knowledge about the law and the description of the circumstances allowed by law on abortion was significantly different when comparing health professionals $(p=0.01)$. When asked about the situations in which Brazilian law allows abortion, $32.7 \%$ of physicians, $97.5 \%$ of nursing professionals and $90.5 \%$ of other professionals were unaware of the law. CONCLUSION: This study demonstrated the lack of of knowledge of Brazilian law among health professionals, to a lesser extent among obstetricians and a to a greater extent among nursing professionals. Attitudes of discrimination and prejudice were observed regarding the care provided to women who induce an abortion.
Correspondêncic

Gláucia Rosana Guerra Benute Avenida Dr. Enéas de Carvalho Aguiar 155 - PAMB - andar téree CEP: 05403-000

São Paulo (SP), Brasil

Recebido $26 / 08 / 2011$

Aceito com modificacões

$13 / 12 / 2011$
Trabalho realizado na Clínica Obstétrica do Hospital das Clínicas da Faculdade de Medicina da Universidade de São Paulo - USP São Paulo (SP), Brasil.

Serviço de Saúde da Divisão de Psicologia do Instituto Central da Faculdade de Medicina da Universidade de São Paulo - USP São Paulo (SP), Brasil.

${ }^{2}$ Divisão de Psicologia e Clínica Obstétrica do Hospital das Clínicas da Faculdade de Medicina da Universidade de São Paulo - USP - São Paulo (SP), Brasil.

${ }^{3}$ Departamento de Obstetrícia e Ginecologia da Faculdade de Medicina da Universidade de São Paulo - USP - São Paulo (SP), Brasil.

${ }^{4}$ Divisão de Psicologia do Hospital das Clínicas da Faculdade de Medicina da Universidade de São Paulo - USP - São Paulo (SP), Brasil.

Departamento de Obstetrícia e Ginecologia do Hospital das Clínicas da Faculdade de Medicina da Universidade de São Paulo USP - São Paulo (SP), Brasil. 
Introdução

O aborto provocado é reconhecido como um problema de saúde pública em todo o mundo, decorrente do alto índice de mortalidade materna associada à sua realização $0^{1,2}$. Dos 20 milhões de abortos inseguros que anualmente ocorrem no mundo com complicações ou sequelas irreversíveis, 97\% acontecem em países em desenvolvimento ${ }^{3}$.

Apesar das restrições legais, das condenações sociais, morais e religiosas e da alta mortalidade materna, o aborto continua existindo, ocasionando $15 \%$ do total das mortes maternas que ocorrem no mundo, por ano ${ }^{4-7}$.

No Brasil, o aborto é responsável por $11,4 \%$ do total de mortes maternas e $17 \%$ das causas obstétricas diretas, com parcela significativa decorrente do aborto provocado ${ }^{6}$. Esse índice pode ser ainda maior, pois não existem estatísticas oficiais e nem estudos com base populacional, dificultando a avaliação da magnitude do problema no país.

O aborto é um tema que está vinculado à vivência reprodutiva da mulher, incluído nas políticas públicas de saúde, nos programas de atenção à saúde da mulher para atendimento no Sistema Único de Saúde (SUS). Está também incluído na formação acadêmica dos profissionais da área da saúde, em especial da medicina e da enfermagem. No entanto, embora faça parte da preparação profissional, a abordagem ainda é influenciada por questões morais, sociais e religiosas que trazem dificuldades para a aceitação do tema e, consequentemente, para a assistência prestada, que acaba sendo norteada pela concepção de que o aborto é um crime, sem referenciar os direitos reprodutivos ou as questões sociais que incluem a problemática da clandestinidade ${ }^{8,9}$.

No Brasil, a legislação acerca do aborto é contemplada no Artigo 128 do Código Penal ${ }^{10}$ de 1940 e exime da condição de crime à interrupção da gravidez quando resultante de estupro (e o aborto é precedido do consentimento da gestante, ou, quando incapaz, de seu representante legal) e em casos de risco de vida à gestante. Nos casos de anomalia fetal incompatível com a vida, embora não esteja contemplado na legislação como aborto legal, a interrupção da gravidez pode ser solicitada caso haja interesse da gestante, mediante autorização judicial.

Considerando que a atitude dos médicos pode ser obstáculo importante para que as mulheres que cumprem os requisitos legais obtenham um aborto em hospital público, Faúndes et al. ${ }^{11}$ realizaram estudo com objetivo de avaliar a opinião e o conhecimento dos médicos sobre o aborto. Concluíram que, de modo geral, tem havido maior reflexão sobre a problemática do aborto, mas evidenciaram a necessidade de informar corretamente os ginecologistas e obstetras sobre as leis e normas que regulamentam a prática do aborto legal no país, visando assegurar que as mulheres tenham, de fato, acesso a esse direito.

Considera-se que a ampliação desse conhecimento para todos os profissionais que atuam em obstetrícia e que lidam de modo direto ou indireto com as mulheres que provocaram o aborto permitirá a adequação do atendimento voltado para a assistência integral à saúde da mulher.

Desse modo, este estudo teve como objetivo verificar o conhecimento acerca da legislação brasileira e da percepção dos profissionais que atuam em obstetrícia relacionadas ao aborto provocado.

\section{Métodos}

Trata-se de estudo prospectivo e transversal. Todos os profissionais que atuam no Departamento de Obstetrícia de um hospital universitário e de um hospital público da periferia de São Paulo (SP) foram convidados a participar. O estudo foi previamente aprovado pelo Comitê de Ética em Pesquisa das instituições envolvidas.

Para a coleta dos dados, foi utilizado um questionário, entregue em envelope fechado. Foi solicitado que os profissionais preenchessem o questionário e depositassem o envelope lacrado em urna específica para tal fim, colocada nos departamentos dos hospitais.

Foram entregues, ao todo, 149 questionários, tendo retornado 119 deles. Para garantir a participação voluntária e anônima, os participantes foram informados de que a devolutiva do questionário respondido seria compreendida como consentimento na participação do estudo.

O questionário continha dados de identificação (idade; profissão; tempo de formação e tempo de trabalho em Obstetrícia), seis perguntas fechadas e uma questão aberta, comum para todos os profissionais entrevistados, além de uma questão fechada específica para médicos.

Dos 119 profissionais entrevistados, 48,7\% eram médicos; 33,6\% eram profissionais da área de enfermagem (auxiliares, técnicos e enfermeiros) e 17,6\% eram profissionais de outras áreas (psicólogos, nutricionistas, fisioterapeutas, administrativos e técnicos de laboratórios).

A média do tempo de formação foi de 139 meses $(\mathrm{DP}=119)$ e a média do tempo de trabalho em Obstetrícia foi de 104 meses $(D P=111)$.

A questão aberta da entrevista foi analisada por meio da Técnica de Análise de Conteúdo ${ }^{12}$. Todos os resultados obtidos com a categorização foram, posteriormente, analisados com técnicas quantitativas por meio do programa Statistical Package for the social Science for Windows (versão 16.0). As variáveis foram analisadas descritivamente, calculando-se médias e desvios padrão, frequências absolutas e relativas. Os dados categóricos e quantitativos 
foram avaliados pelo teste do $\chi^{2}$, sendo adotado nível de significância de 0,05.

Os resultados foram expostos de acordo com a categoria do profissional, dividindo-se entre médicos, profissionais da área de enfermagem e demais profissionais (psicólogos, nutricionistas, fisioterapeutas, administrativos e técnicos de laboratórios).

\section{Resultados}

Quando questionados sobre o que representaria para a sociedade a liberalização do aborto no Brasil, $72,4 \%$ dos médicos, $57,5 \%$ dos profissionais de enfermagem (auxiliares, técnicos e enfermeiros) e 68,4\% dos demais profissionais (psicólogos, nutricionistas, fisioterapeutas, administrativos e técnicos de laboratórios) consideraram um avanço para a sociedade.

A Tabela 1 apresenta os resultados relativos à opinião acerca de em quais situações o profissional acredita que o aborto deveria ser permitido por lei. Constatou-se diferença significativa na proporção de profissionais que acreditam que o aborto por malformação fetal não letal e no aborto decorrente de gestações não planejadas deveriam ser incluídos na legislação brasileira.

$\mathrm{O}$ conhecimento autorreferido acerca da legislação brasileira sobre o aborto e a existência de possibilidades de interrupção da gravidez incluídas na lei encontram-se apresentadas na Tabela 2. Observou-se que a distribuição acerca do conhecimento da legislação e a descrição das situações permitidas por lei acerca do aborto foi significativamente diferente na comparação entre os profissionais de saúde.

Quando questionados sobre as situações em que a legislação brasileira permite o aborto, considerou-se como respostas adequadas aquelas que incluíram risco materno e estupro, assim como aquelas que acrescentavam a solicitação de autorização judicial para os casos de malformação fetal letal. Todas as demais respostas foram consideradas como desconhecimento da legislação vigente.

Quando questionados se acreditam que as pessoas de seu trabalho tratavam as mulheres que provocaram aborto com discriminação, $50 \%$ dos médicos, $7,5 \%$ dos profissionais da enfermagem e $14,3 \%$ dos demais profissionais acreditam que as mulheres que provocaram aborto são tratadas com discriminação. No entanto, a maior parte dos profissionais da enfermagem e dos demais profissionais entregaram o questionário sem responder a essa questão (75 e $80,9 \%$, respectivamente). Com relação ao profissional que observaram a discriminação com relação à mulher que provocou o aborto, tem-se que $88,6 \%$ indicou a categoria médica, $80 \%$ os profissionais da enfermagem e $5,7 \%$ demais profissionais da área de saúde.
Foi solicitado que os médicos apontassem em quais situações eles realizariam, pessoalmente, o aborto, caso a legislação brasileira autorizasse sua realização independentemente do motivo. Foram referidas as situações de estupro $(51,7 \%)$, malformação fetal letal $(62,1 \%)$ e risco materno $(65,5 \%)$.

Tabela 1. Opinião pessoal sobre em quais situaç̃oes 0 aborto deveria ser permitido por lei. Comparaç̃o entre profissionais médicos e não médicos

\begin{tabular}{|c|c|c|c|c|c|c|c|}
\hline \multirow[t]{2}{*}{ Situações de aborto } & \multicolumn{2}{|c|}{ Médico } & \multicolumn{2}{|c|}{ Enfermagem } & \multicolumn{2}{|c|}{$\begin{array}{c}\text { Demais } \\
\text { profissionais }\end{array}$} & \multirow[t]{2}{*}{ Valor $p$} \\
\hline & n & $\%$ & n & $\%$ & n & $\%$ & \\
\hline \multicolumn{8}{|l|}{ Estupro } \\
\hline Sim & 52 & 89,7 & 34 & 85,0 & 20 & 95,2 & 0,4 \\
\hline Não & 6 & 10,3 & 6 & 15,0 & 1 & 4,8 & \\
\hline \multicolumn{8}{|l|}{ Malformação fetal } \\
\hline Sim & 36 & 62,0 & 8 & 20,0 & 5 & 23,8 & 0,01 \\
\hline Não & 22 & 37,9 & 32 & 80,0 & 16 & 76,2 & \\
\hline \multicolumn{8}{|c|}{ Malformação fetal letal } \\
\hline $\operatorname{Sim}$ & 51 & 87,9 & 34 & 85,0 & 20 & 95,2 & 0,4 \\
\hline Não & 7 & 12,1 & 6 & 15,0 & 1 & 4,8 & \\
\hline \multicolumn{8}{|c|}{ Gestação não desejada } \\
\hline $\operatorname{Sim}$ & 31 & 53,4 & 34 & 85,0 & 7 & 33,3 & 0,01 \\
\hline Não & 27 & 46,5 & 6 & 15,0 & 14 & 66,7 & \\
\hline \multicolumn{8}{|l|}{ Risco materno } \\
\hline Sim & 55 & 94,8 & 32 & 80,0 & 18 & 85,7 & 0,07 \\
\hline Não & 3 & 05,2 & 8 & 20,0 & 3 & 14,2 & \\
\hline
\end{tabular}

Tabela 2. Conhecimento da legislação brasileira sobre o aborto: autoavaliação e especificação em quais situações 0 aborto é permitido por lei. Avaliação da pertinência das respostas e comparação dos dados entre profissionais da saúde

\begin{tabular}{|c|c|c|c|c|c|c|c|}
\hline & \multicolumn{2}{|c|}{ Médico } & \multicolumn{2}{|c|}{ Enfermagem } & \multicolumn{2}{|c|}{$\begin{array}{c}\text { Demais } \\
\text { profissioncis }\end{array}$} & \multirow[t]{2}{*}{ Valor $p$} \\
\hline & n & $\%$ & n & $\%$ & $\mathrm{n}$ & $\%$ & \\
\hline \multicolumn{8}{|l|}{$\begin{array}{l}\text { Acredita ter pleno } \\
\text { conhecimento da } \\
\text { legislação? }\end{array}$} \\
\hline Sim & 44 & 75,9 & 17 & 42,5 & 4 & 19,0 & 0,01 \\
\hline Não & 14 & 24,1 & 23 & 57,5 & 17 & 80,9 & \\
\hline \multicolumn{8}{|l|}{$\begin{array}{l}\text { A legislação brasileira } \\
\text { permife a legalização } \\
\text { do aborto em alguma } \\
\text { siłuação específica? }\end{array}$} \\
\hline $\operatorname{Sim}$ & 55 & 94,8 & 33 & 82,5 & 21 & 100,0 & 0,01 \\
\hline Não & 3 & 5,2 & 7 & 17,5 & 0 & - & \\
\hline \multicolumn{8}{|l|}{$\begin{array}{l}\text { Em que situações a } \\
\text { legislação brasileira } \\
\text { permite o aborto? }\end{array}$} \\
\hline Risco materno e estupro & 37 & 63,8 & 1 & 02,5 & 02 & 9,5 & 0,01 \\
\hline $\begin{array}{l}\text { Risco materno, estupro } \\
\text { e autorização judicial } \\
\text { quando MFL }\end{array}$ & 2 & 3,4 & 0 & 00,0 & 0 & - & 0,34 \\
\hline $\begin{array}{l}\text { Desconhece a legislação } \\
\text { vigente }\end{array}$ & 19 & 32,7 & 39 & 97,5 & 19 & 90,5 & 0,01 \\
\hline
\end{tabular}




\section{Discussão}

Os elevados índices de morte materna decorrente do aborto ilegal ${ }^{13}$ fazem necessário que profissionais atuantes na área da saúde se atualizem e repensem constantemente as questões referentes ao aborto.

Os profissionais que atuam em Obstetrícia muitas vezes não conseguem esconder a contrariedade diante da situação do aborto, propiciando assistência pautada no julgamento, na punição, na raiva e na discriminação.

Ao se analisar a percepção de profissionais de enfermagem de uma maternidade pública de Salvador (BA) sobre a assistência prestada à mulher em processo de aborto provocado, constatou-se que esses profissionais entendem o aborto como crime e que a assistência prestada é discriminatória, mesmo nos casos de aborto previstos em lei ${ }^{14}$. Faúndes et al. ${ }^{11}$ apontam que, em 1996, foram evidenciadas as dificuldades enfrentadas por entidades públicas para a implementação de programas de assistência às vítimas de violência sexual, particularmente no que diz respeito à interrupção legal da gestação, por dificuldade de aceitação dos profissionais de saúde.

Em relação à liberalização do aborto, o estudo aponta que a maior parte dos profissionais considerou que o aborto também deveria ser realizado em situações de malformações fetais letais. Nesse estudo, constatou-se que apenas os profissionais médicos consideraram o aborto viável em circunstâncias nas quais a gestação não é planejada ou mesmo em casos de malformações fetais não letais. No entanto, médicos, quando questionados em que situações eles realizariam o aborto, mencionaram as situações já previstas na legislação, incluindo apenas os casos de malformações fetais incompatíveis com a vida extrauterina. Faúndes et al. ${ }^{13}$ observaram que, quando questionados sobre a realização do aborto, $40 \%$ dos médicos mencionaram que ajudariam uma paciente (indicariam um médico de confiança ou ensinariam a usar o misoprostol) que solicitasse o aborto por não desejar a gestação, mas apenas $2 \%$ desse médicos declararam que o realizariam. Entretanto, ressalta-se que quando a questão foi dirigida a situações em que a própria mulher (médica) ou a parceira do homem (médico) estivesse na situação de uma gravidez indesejada, a porcentagem que praticaria o aborto foi de 77,6 e 79,9\%, respectivamente. Nos atendimentos decorrentes do aborto inseguro, o preparo da equipe deve ir além do preparo técnico, estando vinculado às reações emocionais e à maneira de abordá-las. Lunardi e Simões ${ }^{15}$, ao investigarem as reações da equipe de enfermagem frente à possibilidade de participação em aborto legal evidenciaram ausência de conhecimento da legislação diante dos trâmites necessários para sua realização. A avaliação do conhecimento, da opinião e da conduta de obstetras e ginecologistas sobre o aborto induzido e a legislação constatou que $93 \%$ dos médicos responderam corretamente que a lei brasileira não pune o aborto quando a gravidez é resultado de estupro, sendo que $90 \%$ também mencionou os casos de risco de vida materno; porém cerca de um terço respondeu inadequadamente, afirmando que a legislação não penalizava o aborto quando o feto fosse acometido por malformações graves ${ }^{13}$.

Esse estudo constatou deficiência inegável por parte dos profissionais da saúde quanto ao conhecimento dos preceitos legais que regulam o aborto no Brasil, dificultando o cumprimento da lei em hospitais e serviços de saúde e interferindo na qualidade do atendimento prestado às pacientes que abortam.

A competência técnica dos profissionais é ameaçada quando existe dificuldade de interação com as pacientes, principalmente quando os problemas de saúde são estigmatizados dentro da sociedade ${ }^{16}$. Assim, o preconceito e a interferência de crenças pessoais no tratamento das pacientes possibilitam que o atendimento seja realizado em função das necessidades físicas, negligenciando o suporte emocional ou mesmo orientações educativas ${ }^{17,18}$. Nery et al. ${ }^{19}$ aponta que, nas situações de aborto, são relevantes para as mulheres que o vivenciaram a afetividade e o interesse dos profissionais vinculados aos procedimentos técnicos ${ }^{16}$, pois tal conduta tem repercussões positivas no estado emocional das pacientes.

Para que a assistência integral à saúde da mulher, tal como proposta no SUS, possa ocorrer, torna-se de fundamental relevância que os profissionais estejam integrados tanto com os aspectos técnicos, éticos e jurídicos do aborto, quanto com seus aspectos subjetivos, evitando o julgamento, o preconceito e propiciando mais do que a técnica, a interação, a afetividade, o acolhimento. Neste estudo, evidenciou-se o desconhecimento dos profissionais de saúde com relação à legislação brasileira, em proporção inferior entre obstetras e em proporção superior entre os profissionais da área de enfermagem. 
1. Gasman N, Blandon MM, Crane BB. Abortion, social inequity, and women's health: obstetrician-gynecologists as agents of change. Int J Gynaecol Obstet. 2006;94(3):310-6.

2. Hessini L, Brookman-Amissah E, Crane BB. Global policy change and women's access to safe abortion: the impact of the World Health Organization's guidance in Africa. Afr J Reprod Health. 2006; 10(3): 14-27.

3. Grimes DA, Benson J, Singh S, Romero M, Ganatra B, Okonofua $\mathrm{FE}$, et al. Unsafe abortion: the preventable pandemic. Lancet. 2006;368(9550): 1908-19.

4. Sedgh G, Henshaw S, Singh S, Ahman E, Shah IH. Induced abortion: estimated rates and trends worldwide. Lancet. 2007;370(9595):1338-45.

5. Guttmacher Institute [Internet]. Facts on induced abortion worldwide. 2011 Feb [cited 2011 Jun 10]. Available from: http://www. guttmacher.org/pubs/fb_IAW.pdf

6. Brasil. Ministério da Saúde [Internet]. Norma técnica humaniza atendimento às mulheres com complicações de abortamento. 2001 [cited 2011 Jun 10]. Available from: http://portal.saude. gov.br/saude/visualizar_texto.cfm?idtxt=22411

7. Olinto MTA, Moreira-Filho DC. Fatores de risco e preditores para o aborto induzido: estudo de base populacional. Cad Saúde Pública. 2006;22(2):365-75.

8. Soares GS. Profissionais de saúde frente ao aborto legal no Brasil: desafios, conflitos e significados. Cad Saúde Pública. 2003;19 Suppl 2:399-406.

9. Colas $O$, Aquino NMR, Mattar R. Ainda sobre o aborto legal no Brasil e o conhecimento dos profissionais de saúde. Rev Bras Ginecol Obstet. 2007;29(9):443-5.
10. Brasil. Decreto-Lei n. 2848 de 7 de dezembro de 1940. Código penal. 1940 [Internet]. [cited 2011 Jun 10]. Available from: http://www. tesouro.fazenda.gov.br/gfm/legislacao/Dec_Lei2848_1940.pdf

11. Faúndes A, Duarte GA, Osis MD, Andalaft-Neto J. Variações no conhecimento e nas opiniões dos ginecologistas e obstetras brasileiros sobre o aborto legal, entre 2003 e 2005. Rev Bras Ginecol Obstet. 2007;29(4):192-9.

12. Bardin L. Análise de conteúdo. 5a ed. Lisboa: Edições 70; 2009.

13. Faúndes A, Duarte GA, Andalaft-Neto J, Olivatto AE, Simoneti RM. Conhecimento, opinião e conduta de ginecologistas e obstetras brasileiros sobre o aborto induzido. Rev Bras Ginecol Obstet. 2004;26(2):89-96.

14. Gesteira SMA, Diniz NMF, Oliveira EM. Assistência à mulher em processo de abortamento provocado: discurso de profissionais de enfermagem. Acta Paul Enferm. 2008;21 (3):449-53.

15. Lunardi VL, Simões AR. (Re)Ações da equipe de enfermagem frente à possibilidade de participação em um aborto legal. Rev Enferm UERJ. 2004;12(2):173-8.

16. Motta IS. A relação interpessoal entre profissionais de saúde e a mulher em abortamento incompleto: "o olhar da mulher". Rev Bras Saúde Matern Infant. 2005;5(2):219-27.

17. Ribeiro-Neto JJA. Profissional de saúde e o aborto: é preciso humanizar o atendimento. J Rede Fem Saúde. 2000;(21):6-8.

18. Duarte DA, Melo-Almeida MG. Conhecimento dos profissionais de saúde frente ao aborto legal no Brasil: uma revisão bibliográfica. Rev Baiana Saúde Pública. 2010;34(2):279-87.

19. Nery IS, Monteiro CFS, Luz MHBA, Crizóstomo CD. Vivências de mulheres em situação de aborto espontâneo. Rev Enferm UER. $2006 ; 14(1): 67-73$. 